\title{
Cooperation among cancer cells as public goods games on Voronoi networks
}

Marco Archetti

School of Biological Sciences, University of East Anglia, Norwich Research Park, Norwich NR4 7TJ, UK.

Email: m.archetti@uea.ac.uk

\begin{abstract}
Cancer cells produce growth factors that diffuse and sustain tumor proliferation, a form of cooperation among cancer cells that can be studied using mathematical models of public goods in the framework of evolutionary game theory. Cell populations, however, form heterogeneous networks that cannot be described by regular lattices or scale-free networks, the types of graphs generally used in the study of cooperation. To describe the dynamics of growth factor production in populations of cancer cells, I study public goods games on Voronoi networks, using a range of non-linear benefits that account for the known properties of growth factors, and different types of diffusion gradients. The results are surprisingly similar to those obtained on regular graphs and different from results on scale-free networks, revealing that network heterogeneity per se does not promote cooperation when public goods diffuse beyond one-step neighbours. The exact shape of the diffusion gradient is not crucial, however, whereas the type of non-linear benefit is an essential determinant of the dynamics. Public goods games on Voronoi networks can shed light on intra-tumor heterogeneity, the evolution of resistance to therapies that target growth factors, and new types of cell therapy.
\end{abstract}

Keywords: Game Theory; Cancer; Growth Factors; Cooperation; Diffusion; Voronoi. 


\section{Introduction}

\section{Growth factors as public goods}

At least five of the hallmarks of cancer [Hanahan \& Weinberg 2011] depend on the production of diffusible factors by cancer cells [Witsch et al. 2010]: self sufficiency in growth signals, evading apoptosis, sustained angiogenesis, immune system evasion and the initiation of metastases. Because most of the cytokines and growth factors that promote these processes diffuse in the extra-cellular matrix, their effect is not limited to the cells that produce them, and non-producing cells can use the factors diffusing from neighbouring producer cells. The production of such diffusible factor is, in other words, a form of cooperation between cancer cells [Jouanneau et al. 1994, Axelrod et al. 2006, Archetti et al. 2013a] and growth factors are a type of public good (the term "common goods" would be more appropriate because, "public goods" are often defined as being non-rivalrous, as well as non-excludable [Samuelson 1954]; this definition, however, applies to very few cases, and "public good" has been used more often in biology).

\section{Public goods in evolutionary game theory}

Public goods raise a collective action problem: why contribute to the production of a public good instead of free-riding on the goods (in our case growth factors) produced by other group members? The overexploitation of common-pool resources is a common outcome of such collective action problems [Hardin 1965]. Because of the strategic, frequency-dependent nature of the interactions, the most appropriate framework for the study of public goods is game theory. Evolutionary game theory [Maynard Smith 1982], in particular, is appropriate because it does not assume rational behaviour; instead, the individuals (or cells), programmed to take the best decision have higher fitness and increase in frequency in the population by natural (clonal) selection. The study of cooperation has a long tradition in biology [Nowak 2006]. Public goods games in biology have been reviewed recently [for well-mixed populations: Archetti \& Scheuring 2012; for spatially structured populations: Perc et al. 2013].

\section{Growth factors are non-linear public goods}

For the study of growth factor production by cancer cells we must use assumptions that are rarely used in the study of public goods games. First, models of public goods often assume that fitness is a linear function of number of producers. For cancer cells this would imply that proliferation is a linear function of the amount of circulating growth factors, an assumption that is clearly not true; growth rates of cancer cells are typically a sigmoid function of the concentration of growth factors [e.g.: Valenzano et al. 1997, Karey \& Sirbasku 1988, Jourdan et al. 2005]. We will assume different types of non-linear benefits here.

\section{Decoupling the update and interaction neighborhood}

Another standard assumption in the study of public goods is that an individual can affects only the fitness of its one-step neighbours; more specifically, the assumption is that an individual belongs to multiple groups, each group centred on one of that individual's one-step neighbours, and that individual's fitness is the sum of all the payoffs accumulated by that individual in all these groups [Perc et al. 2013]. While this assumption is reasonable for human interactions in social networks, growth factors typically diffuse beyond a cell's one-step neighbours. We must assume, therefore, that the payoff 
for an individual is a function of the number of producers within the diffusion range of the growth factor, which defines the interaction group. In other words, in order to study diffusible public goods, we must decouple the interaction neighborhood (the group playing the public goods game) and the update neighborhood (the one-step neighbors). This approach has been used to study a two-person game with a linear benefit function (the Prisoner's Dilemma) on a regular lattice [Ifti et al. 2004, Ohtsuki et al. 2007] and for non-linear public goods games on regular lattices with a fixed diffusion range [Archetti $2013 b]$.

\section{Diffusion gradients}

Given that growth factors diffuse beyond one-step neighbours, we must define the shape of the diffusion gradient. Because public goods games have typically assumed a one-step diffusion range, there has been little scope for analysing diffusion gradients so far. Four recent studies [Allen et al. 2013, Borenstein et al. 2013, Scheuring 2013, Archetti 2013b] have analysed diffusion gradients in the context of public goods, and have reached rather discordant conclusions because they used different assumptions, particularly on the shape of the benefit function. If the benefit function is non-linear, the shape of the diffusion gradient seems to be largely irrelevant for the dynamics, and can be approximated by a step function [Archetti 2014a]. This result may be limited, however, to the type of graphs (regular lattices) used in those studies.

\section{Voronoi networks}

The main departure from the standard approach analysed here is the use of Voronoi networks. Two topologies are usually considered in the study of spatial games: regular lattices, in which all individual nodes are topologically equivalent, and scale-free networks, in which different individuals have a distinct number of connections [reviewed in Perc et al. 2013]. While regular lattices neglect the importance of variation in connectivity, scale-free networks are not appropriate if players are distributed on a planar network. The distribution of cells in biological tissues resembles a Voronoi diagram [Lewis 1928, Honda 1978, Gibson \& Gibson 2009, Csikász-Nagy et al. 2013] rather than a regular lattice or a scale-free network.

A Voronoi diagram (tessellation) of a set of nodes is a collection of convex polygons, each corresponding to one of the nodes, with all the points in one polygons being closer to the corresponding node than to any other node; the boundary between two adjacent polygons is a line segment, and the line that contains it is the perpendicular bisector of the segment joining the two nodes. A Voronoi network is defined as a group of such node-joining segments [Figure 1]. The average connectivity of Voronoi networks is 6 , with a unimodal distribution in which fewer than 4 or more than 8 connections are very rare - similar to what is observed in biological samples, both within and among species [Lewis 1928, Gibson \& Gibson 2009, Csikász-Nagy et al. 2013].

Voronoi tesselations have been used [Rejniak \& Anderson 2011, Csikász-Nagy et al. 2013] to simulate the growth of multicellular spheroids [Schaller \& Meyer-Hermann 2005, Beyer \& MeyerHermann 2009] and the development of colorectal tumors [Meineke et al. 2001, Van Leeuwen et al. 2009], but not in the study of cooperation between cancer cells, or, more in general, in evolutionary game theory. A preliminary analysis of cooperation on Voronoi networks with a fixed diffusion range has been included in a recent experimental study of IGF-II production in pancreatic cancer [Archetti et al. 2015]. Here I describe in detail the effect of the parameters on the dynamics of non-linear public goods games on Voronoi graphs, using different types of diffusion gradients, and I compare the results with the standard approach on regular lattices and scale-free networks. 


\section{Methods}

\section{Game structure}

Individual cells occupy the nodes of a network (population size $Z$ is fixed at 1000) and interactions proceed along the edges connecting the nodes, as is standard in public goods game. In the standard approach, however, a node participates in all public goods games defined by the groups the node belongs to (that is, the groups centred on the node's one-step neighbours), and the node's fitness is given by the sum of the payoffs accumulated by the individual at that node in these games [Perc et al. 2013]. Here, instead, individual cells participate in only one game, in the group defined by the diffusion range of the growth factor. In the simplest scenario, with a fixed diffusion range, the group is simply defined by the number of edges $d$ between the focal node and the most distant node whose contribution affects the fitness of the focal node [Figure 1]. When we assume a diffusion gradient we will make more specific assumptions on the shape of this gradient and how it affects fitness (see below).

\section{Strategies and payoffs}

A cell can be a producer or a non-producer of a growth factor. All cells benefit from the effect of the growth factors produced by cells in their group. The payoff for a cell is $b(j)-c$, where $c$ is the cost of producing the growth factor $(0<c<1$ for producer cells and $c=0$ for non-producer cells $), j$ is the number of producer cells in the group and

$b(j)=[V(j)-V(0)] /[V(n)-V(0)]$

is the normalized version of the logistic function

$V(j)=1 /\left[1+e^{-s(j-k) / n}\right]$

that describes the non-linear effect of the concentration of the growth factor as a function of the number of producer cells in the group. Group size $n$ depends on the diffusion range or gradient of the growth factor. The parameter $k$ controls the position of the inflection point $(k \rightarrow n$ gives strictly increasing returns and $k \rightarrow 0$ strictly diminishing returns) and the parameter $s$ controls the steepness of the function at the inflection point $(s \rightarrow \infty$ models a threshold public goods game; $s \rightarrow 0$ models an Nperson prisoner's dilemma) [Archetti \& Scheuring 2011, 2012].

\section{Update rules}

The process starts with a number of non-producers placed at random on the graph; unless stated otherwise, the initial fraction of non-producers is 0.05 . At each round, strategies are updated according to the following rule: a node $x$ with payoff $\mathrm{P}_{x}$ is selected (at random) for update (death); a node $y$ (with payoff $\mathrm{P}_{y}$ ) is then chosen among $x$ 's neighbors. Two types of update are used: in the deterministic case, if $\mathrm{P}_{x}>\mathrm{P}_{y}$, no update occurs, while if $\mathrm{P}_{x}<\mathrm{P}_{y}, x$ will adopt $y$ 's strategy (unconditional imitation); in the stochastic case, replacement occurs with a probability given by $\left(\mathrm{P}_{\mathrm{y}}-\mathrm{P}_{\mathrm{x}}\right) / \mathrm{M}$, where $\mathrm{M}$ ensures the proper normalization and is given by the maximum possible difference between the payoffs of $x$ and $y$ [Perc et al. 2013]. Results are obtained averaging the final 200 thousand of 1 million generations (that is, the final 200 of 1000 replications per cell), averaged over 10 different runs. 
Diffusion gradient

While in models with a fixed diffusion range a cell's payoff is determined by the number of producer cells within a range $d$ from that cell, in a model with a diffusion gradient each cell receives contributions from other producer cells within a diffusion range $D$ of the molecule, and each contribution is weighted ccording to the distance $i$ (the number of nodes) from that cell according to the function [Archetti 2014a]

$G(i)=1-[g(i)-g(0)] /[g(D)-g(0)]$

where

$g(i)=1 /\left[1+e^{-z(i-d) / D}\right]$

The values of $d$ and $D$ determine the shape of the diffusion gradient, which is always decreasing but can be concave $(d=D)$, convex $(d<<D)$ or sigmoid (intermediate values of $d<D)$. In the computation of payoffs, each producer cell contributes $G(i)$, instead of 1 , to the number of producers in the group. The number of producers, therefore is not $j$ but the weighted sum of all $G(i)$ values. For example, if $d=D / 2$ and there are six producer cells all $d$ nodes away from a focal cell ( $i=d$ for all producers), $G(d)=1 / 2$, that is, only half of each producer's contribution is available for the focal cell, hence the weighted number of producers (the sum of all the contributions) in that focal cell's group is three. With a more realistic distribution of $i$ values, the calculation is more complex. The parameter $z$ controls the steepness of the gradient at the inflection point: $z \rightarrow 0$ models a linear gradient; $z \rightarrow \infty$ models a step function equivalent to the case a fixed range (the diffusion range is a step function).

\section{Topology}

The Voronoi diagram of a set of vertices $\mathrm{V}$ is a subdivision of space into Voronoi cells; for any vertex $i$ belonging to $\mathrm{V}$, the Voronoi cell of $i$ is the set of points with distance to $i$ not greater than to any other vertex of $\mathrm{V}$. The dual of the Voronoi diagram is the Delaunay triangulation defined on the same vertex set (Voronoi polygons correspond to Delaunay vertices) [Figure 1]. The two-dimensional Voronoi graphs used here are obtained by a Delaunay triangulation of random points on a sphere, using the DelaunayTriangulation implementation in Mathematica 8 (Wolfram Research Inc.). If the points are drawn on a circle or on a square, the process is straightforward [Figure 1b-c]. Points can be also drawn on a sphere in order to avoid edge effects (this is equivalent to the common procedure of connecting the edges of a regular lattice to form a toroidal network). Points on a sphere are defined by colatitude $(\phi)$ and longitude $(\theta)$ both drawn from a uniform distribution with support $[0,2 \pi]$. These can be mapped into the Cartesian space using the standard transformation

$x=r^{*} \sin \phi \cos \theta$

$y=r^{*} \sin \phi \sin \theta$

$z=r^{*} \cos \phi$

with $r=1$. With this simple mapping, however, the points are more dense at the poles. Because the Jacobian determinant of the mapping (which describes how the spherical space is modified by the transformation at each point) is independent of $\theta$ our uniform distribution in spherical coordinates is uniform in $\theta$ ( $\theta$ is drawn from $[0,2 \pi])$, but not in $\phi$. Instead of drawing $\phi$ from $[0,2 \pi]$ therefore we 
define it as $2 \mathrm{ArcSin}[\operatorname{Sqrt}[\operatorname{Random}[0,1]]$ using the "Inverse CDF Method" in Mathematica. This reduces the density of points around the poles, making them evenly distributed over the spherical area [Figure 1d]. In the Cartesian space the polygons appears distorted ad the edges, and they are actually connected with the polygons on the opposite edge to form a sphere [Figure 1e].

Regular graphs with connectivity 6 are obtained by a modification of the GridGraph implementation in Mathematica and connecting opposing edges to form a toroidal network, in order to avoid edge effects. Scale-free networks are obtained by means of the Barabasi-Albert model based on growth and preferential attachment [Barabasi \& Albert 1999], using the BarabasiAlbert GraphDistribution implementation in Mathematica.

\section{Results}

\section{Differences with the standard approach}

Since the average connectivity on a Voronoi graph is 6, it is useful to compare the results to those obtained on regular lattices with connectivity 6 . It is also useful to compare results on scale-free networks, and results obtained using the standard approach (no diffusion beyond one-step neighbours, payoffs calculated over multiple games) [Figure 2].

In the standard approach, the cost of public good production $(c)$ is the main determinant of the type of dynamics: if $c$ is small enough, a mixed equilibrium exists in which producers and nonproducers persist; the fraction of producers increases with $h$ (the position of the inflection point in the benefit function). Scale-free network lead to cooperation for higher costs when $b$ is low. In other words, scale-free networks seem to promote cooperation, compared to regular lattices and Voronoi networks. Regular lattices allow cooperation for slightly higher values of $c$ at intermediate values of $h$, compared to Voronoi networks, but there are no other significant differences between regular lattices and Voronoi networks [Figure 2].

With diffusible public goods (diffusion beyond one-step neighbours), scale-free networks lead to cooperation only for low values of $c$, even though they allow cooperation for higher and lower $b$ values, and the level of cooperation is lower, compared to planar networks. Regular lattices allow cooperation for slightly higher values of $c$ compared to Voronoi networks, again, with no other significant differences [Figure 2]. In summary, the results with regular graphs and Voronoi graphs are remarkably similar, whereas scale-free networks lead to very different results when diffusion goes beyond one-step neighbours. Voronoi graphs seem to be less conducive to cooperation than regular lattices.

These differences in the amount of cooperation at equilibrium may be explained, at least in part, by differences in group size $n$ [Figure 3]. It is well known that mixed equilibria in non-linear public goods games exist only below a critical value of $c$ or $n$ and that the amount of cooperation declines with $c$ and $n$. The update neighborhood on a Voronoi graph has on average the same size as on a regular lattice with connectivity 6. Voronoi graphs, however, tend to have larger groups (interaction neighborhoods) on average as $d$ increases, and obviously higher variance in group size. Scale-free networks have an even larger variance, with many nodes with small connectivity and very few nodes with much higher connectivity. [Figure 3]. The average size of the interaction neighborhood for $d=2$ is $n=19$ for a regular lattice with connectivity 6 , it is $n=20$ for a Voronoi network and $n=70$ for a scale-free network; for $d=5$ it is $n=91$ for a regular lattice with connectivity $6, n=109$ for a Voronoi network and $n$ is at least two orders of magnitude higher for a scale-free network.

In summary, the higher levels of cooperation observed on planar networks (regular lattices and Voronoi networks) seems to be due simply to the fact that, when public goods diffuse beyond one-step neighbours, average group size (and variance) is much higher in scale-free networks. The small 
difference observed between regular lattices and Voronoi networks is due in part to the fact that Voronoi graphs have groups with variable size, which is on average slightly higher than in regular lattices.

\section{Effect of costs and benefits}

The fact that the production of public goods is more efficient in smaller groups is well known and may explain the differences in the level of cooperation attained in different graph types, as mentioned above. For the same reason, since group size increases with the diffusion range of the growth factor (d), we also expect lower levels of cooperation as $d$ increases. This is indeed what is observed [Figure 4]. Again, regular lattices allow slightly higher levels of cooperation than Voronoi graphs. Cooperation cannot be maintained on scale-free networks if $c$ is not negligible and $d$ is higher than 2 . This is again arguably an effect of group size. For $d=3$ group size is $n=37$ for a regular lattice with connectivity $6, n=42$ on average for a Voronoi network and $n>300$ for a scale-free networks. For $d=5$, group size is still $n=91$ for a regular lattice, and $n=109$ for a Voronoi network, but at least two orders of magnitude higher for a scale-free network. While scale-free networks can promote cooperation in the standard approach [Perc et al. 2013], therefore, they do not lead to any cooperation at all when public goods diffuse beyond one-step neighbours, simply because group size increases rapidly with $d$.

The amount of cooperation declines with $c$ (the cost of public good production) and $s$ (the steepness of the benefit function) on Voronoi networks [Figure 5]. As expected, a stochastic update rule is also less conducive to cooperation.

Analysing how the structure of the producer and non-producer clusters evolve [Figure 6], reveals that non-producers form clusters whose size increases with $d$. The average degree centrality (the number of neighbours) and the closeness centrality (the shortest distance between all pairs of nodes) of the producer and non-producer subgraphs are relatively constant (apart from an initial adjustment period, especially for high $d$ ). The non-producer subgraphs have generally a lower average degree centrality and a higher average closeness centrality; that is, non-producers form small and narrow clusters among the producers, unless the diffusion range is large, in which case the centrality measures are similar.

In summary the dynamics and equilibria of public goods games on Voronoi networks are not radically different from the results observed in regular lattices. Node heterogeneity does not promote cooperation (as in scale-free networks in the standard approach) and does not alter radically the dynamics.

\section{Effect of the diffusion gradient}

The results above assume a fixed diffusion range (a cell's payoff is determined by the number of producer cells within a range $d$ from that cell). If growth factors diffuse according to a more realistic gradient, results change slightly [Figure 7]. Different types of diffusion gradient lead to higher levels of cooperation. More specifically, while the critical value of $c$ that allows a mixed equilibrium remains the same for intermediate values of $h$, at low and high values of $h$ cooperation is possible at higher values of $c$ when we assume a diffusion gradient.

While a concave (upwards) diffusion gradient seems to lead to a higher level of cooperation than a linear or sigmoid gradient, this may be due simply to a lower weighted number of cells. The overall result (a mixed equilibrium in which producers decline in frequency with $c$ and for extreme values of $h$ ) seems consistent across different types of gradient.

Diffusion gradients lead to slightly lower degree centrality and slightly higher closeness centrality for the non-producer subgraph, that is, slightly smaller and narrower clusters of non- 
producers among the producers [Figure 8]. Again, this may be due to a smaller effective group size [Figure 6].

\section{Perturbation of the inflection point}

Anti-cancer therapies that reduce the amount of circulating growth factors can reduce tumor fitness in the short term because immediately after treatment the amount of growth factor produced by the (pretreatment) equilibrium fraction of producer cells is no longer enough to sustain tumor growth [Figure 9]. The cell population, however, is no longer at an equilibrium after the therapy, because removing part of the circulating growth factors increases the amount of factors that the cells must produce to achieve the same pre-treatment benefit, that is, it increases the value of the inflection point $h$. The population therefore will adapt to the new $h$ value, reaching a new equilibrium. Since the post-treatment threshold $(b)$ is higher, this can eventually lead to an even higher growth rate for the tumour at the new equilibrium [Figure 9]. Both the magnitude of the change and its speed are crucial for the success of the therapy: a large change is more likely to lead to a lower tumour fitness, a slow transition can make even a very effective therapy to fail and lead to relapse [Figure 10].

\section{Summary of the results}

In summary, the results of public goods games on Voronoi networks are similar to those obtained on regular graphs with the same average connectivity. Voronoi graphs are slightly less conducive to cooperation than regular lattices because group size is variable and, on average, slightly higher on Voronoi networks. Scale-free networks, on the other hand, lead to very different results, and when diffusion goes beyond one-step neighbours they do not allow the maintenance of cooperation. Node heterogeneity is not crucial in determining the dynamics: the existence of a mixed equilibrium (coexistence of producers and non-producers) and the amount of cooperation attained depend mainly on the diffusion range of the public good (high diffusion reduces cooperation), on the shape of the benefit function and on the cost of producing the public good. Like in well-mixed populations and in regular lattices, cooperation declines with the cost of public good production and with the steepness of the benefit function, and when a stochastic update rule is adopted. While diffusion gradients lead to higher levels of cooperation, the exact shape of the gradient does not have a significant effect on the dynamics.

\section{Discussion}

Network heterogeneity does not promote cooperation for diffusible public goods

Heterogeneity in the number of neighbours promotes cooperation in standard public goods games because, essentially, it leads to a heterogeneous payoff distribution, which reduces the critical value of the benefit necessary for cooperation at nodes with low connectivity [Perc et al. 2013]. This conclusion, however, is based on results for non diffusible public goods (and assumes linear benefits on scale-free networks). In that case the difference between the payoff of a producer and a non-producer is inversely proportional to the number of games each player plays, that is to the number of neighbours. In the case of diffusible public goods analysed here, instead, each player plays one game only, and only group size varies. Moreover, other properties besides degree distribution can be important for the dynamics of public goods, such as the average path length [Szabó \& Fáth 2007], the clustering coefficient [Rong et al. 2010] or the presence of correlations among high-degree nodes [Rong \& $\mathrm{Wu}$ 2009]. Results obtained on scale-free networks, therefore, are not necessarily relevant for social dilemmas on Voronoi 
networks. As we have seen here, indeed, the heterogeneity of Voronoi networks does not enhance cooperation compared to regular lattices. A slightly lower level of cooperation is actually observed on Voronoi networks, which seems to be due essentially to the fact that group size is slightly higher, on average, compared to regular lattices.

\section{Diffusion matters, but the exact shape of the diffusion gradient does not}

Diffusion beyond one-step neighbours is an essential assumption in the case of growth factors. While diffusion can be modeled simply by assuming that a cell's payoff is determined by the number of producer cells within a fixed range from that cell, it could be argued that a more realistic diffusion gradient, in which the effect or concentration of the growth factor declines gradually from the source, can have important effects on the dynamics. As we have seen, however, the exact shape of the diffusion gradient is not crucial, and the amount of cooperation attained can be approximated (if slightly underestimated) using a model with a fixed diffusion range. While the effect of diffusion gradients is stronger on Voronoi networks than on regular lattices, where diffusion gradients have a negligible effect on cooperation [Archetti 2014a], even on Voronoi graphs using different types of diffusion gradient leads to similar results. In summary, while assuming diffusion beyond one-step neighbours is crucial, exact assumptions on the shape of the diffusion gradient are not.

\section{The type of non-linear benefit is the main determinant of the dynamics}

The main determinant of the dynamics if the type of benefit function. While evolutionary game theory has traditionally focused on linear benefits, biological molecule, have rarely, if ever, linear effects and the effect of a growth factor on cell proliferation is generally a sigmoid function of its concentration [Cornish-Bowden 2012], because the series of reactions produced by the cascade of signal originating from the cellular receptors amplifies even the slightest departure from linearity of the underlying individual chemical reactions [Frank 2013]; sigmoid functions are produced, for instance, by positive cooperative binding, by titration of repressors or by opposing saturated forward and back reactions [Zhang et al 2013]. The effect of growth factors on cell proliferation has been reported to be a sigmoid function in different types of cancer [e.g.: Valenzano et al. 1997, Karey \& Sirbasku 1988, Jourdan et al. 2005]. Non-linearities play a major role in the dynamics of diffusible public goods. If the benefit of the molecule is a linear function of its concentration, either producers or non-producers have a higher fitness for any frequency of producers, depending only on the relative cost/benefit of producing the molecule (in sizeable groups, for reasonable costs producers will always have a disadvantage, this results in what we usually refer to as "N-person Prisoner's Dilemma"). If benefits are non-linear, however, whether producers or non-producers have higher fitness depends on the frequency of the two types; if the cost is not too high, a stable polymorphism is possible [Archetti \& Scheuring 2011, 2012]. Steep benefit functions allow stable polymorphic equilibria for larger costs, but they make the population less robust to random fluctuations in the fraction of producers. Differently from the case of well-mixed populations, a very steep benefit function makes it profitable to contribute if and only if that contribution is pivotal for the production of the public good, that is, only when there are exactly $k-1$ other producers. In spatially structured populations it can happen that a non-producer mutant arises in a group centred on one individual with few non-producers, and therefore can invade that group; this can however lead an adjacent group that was previously at equilibrium, with exactly $k$ contributors, below the unstable equilibrium, which ultimately leads to the extinction of producers in that group [Archetti 2014]. Node heterogeneity in Voronoi networks does not seem to change this result. 
The dynamics depends on the cost/benefit ratio of the growth factor $c / b$, its diffusion range $d$ and the shape of the benefit function, that is its inflection point $b$ and steepness $s$. Knowing the value of these parameters would allow us to predict the dynamics of a population of cells.

The $c / b$ ratio can be estimated by comparing the growth rates of pure producer and nonproducer populations. By providing a saturating amount of exogenous growth factor the difference in growth between producers and non-producers essentially measures the cost of producing the growth factor (in pancreatic cancer, $c / b$ can be estimated to be approximately 0.2 [Archetti et al. 2015]).

The steepness of the benefit function $(s)$ can be estimated by the dose-response curve of the growth factor, that is, by measuring cell proliferation as a function of growth factor density in the growth medium. In order to estimate the inflection point $h$, instead, one should measure the benefit as function of the fraction of producers rather than of the amount of growth factor; while this could be estimated indirectly by knowing the amount of growth factor produced by a single cell, a direct measurement requires measuring the growth rates of populations with different fractions of producers.

The diffusion range, on the other hand, is more difficult to measure, and is not know for most growth factors. The diffusion of EGF in the brain has been estimated to be $5 \times 10^{-7} \mathrm{~cm}^{2} / \mathrm{s}$ [Thorne et al. 2004] which is equivalent to approximately $0.05 \mathrm{~cm} /$ hour; therefore $d$ can be estimated to be approximately 25 to 50 if we assume that the size of a cell is $10-20 \mu \mathrm{m}$. Thorne et al. 2004 actually calculate the diffusion range of EGF; using the curve shown in their Fig. 6, the diffusion range at which the concentration of the growth factor is $50 \%$ of the concentration at the source is between 100 and $600 \mu \mathrm{m}$, that is (assuming again that cell size is between 10 and $20 \mu \mathrm{m}$ ), $d=5-10$ to 30-60. Clearly however, diffusion depends on the half-life of the growth factor and on cell type, and should be measured for each combination of growth factor and tumor type (although it is possible that diffusion may change in a systematic way based on cell size and the size of the growth factor, which would enable to estimate the diffusion range based on growth factor and tissue of origin).

\section{Implications for cancer research}

Growth factor production by cancer cells is essential for tumor development. Tumors grow and expand when mutations enable cancer cells to produce their own growth factors (or lead to the constitutive activation of their receptors or downstream pathway) that promote proliferation, allow to evade apoptosis and immune system reaction, and promote neo-angiogeneis. Metastases also depend on the production of growth factors that induce the epithelial-mesenchimal transition. Understanding the dynamics of the collective interactions that regulate growth factors, therefore, is important for our understanding of tumor growth. More specifically, the frequency-dependent nature of the interactions described by evolutionary game theory can shed light on the maintenance of intra-tumor heterogeneity, on the development of resistance to therapies, and on the development of new cell therapy approaches.

Intra-tumor heterogeneity, the coexistence of different clones within a tumor, is commonly observed [Greenman et al. 2007, Navin et al. 2011] and has implications for cancer progression, diagnosis, and treatment [Dexter \& Leith 1986, Maley et al. 2006, Almendro et al. 2013]. Why such heterogeneity exists remains unclear [Merlo et al. 2006, Almendro et al. 2013], because clones that have a proliferative advantage within the tumour are expected to drive other subclones to extinction. In the light of evolutionary game theory, however, stable intra-tumor heterogeneity is easily explained as a stable polymorphic equilibrium resulting from the non-linear benefit of growth factors [Archetti et al. 2015]. On Voronoi networks this intra-tumor heterogeneity has the same origin: the frequencydependent dynamics leads to a stable coexistence of producers and non-producers. 
Effect on the analysis of therapies based on growth factors

The effect of modern therapies that target growth factors (or their receptors) can be studied as the perturbation of the parameters of a public goods game, namely, as we have seen, perturbations of the inflection point of the benefit function. It has been suggested that treatments that target growth factors may be less susceptible than traditional drugs to the evolution of resistance [Pepper 2012, Aktipis \& Nesse 2013]. An analysis of the perturbation of the equilibria of the system can simulate the effect of therapies that target growth factor, and reveal their stability and efficacy. Because the effect of growth factors is generally a non-linear function of its concentration, treatments that target growth factors need to be extremely efficient and fast in order to be evolutionarily stable both in well-mixed populations [Archetti 2013a] and in regular graphs [Archetti 2013b].

While in well-mixed populations and in regular graphs group size is the same for all groups, and therefore changing the threshold $b$ affects all groups equally, in Voronoi graphs group size is variable, and as a consequence changing the threshold $h$ (that is, introducing a therapy that targets growth factors) could move some clusters of cells in the basin of attraction of the pure non-producer type while keeping other clusters in the basin of attraction of the mixed equilibrium. This makes it more likely for producers to go extinct. Indeed, as we have seen, network heterogeneity may lead to less cooperation than regular graphs with the same average connectivity. The overall, qualitative effect of targeted therapies, however, is the same on Voronoi and regular graphs: reducing the amount of circulating growth factors increases the value of $h$, changing the dynamics and leading to a new equilibrium with a higher fraction of producers (hence a higher growth rate that may lead to relapse) unless the change is large enough or fast enough.

Finally, the long term dynamics of cell therapies that rely on genetically modified cancer cells, engineered to knock-out growth factors, can also be analysed using public goods games on Voronoi networks. Again, like for the study of resistance to therapies, node heterogeneity, may affect the dynamics. As the dynamics of such therapies depends on the the group size at the edge of clusters of producers and non-producer cells [Archetti 2013c], the higher variance of group size in Voronoi networks could make a difference.

\section{Further developments of the model}

While it is now understood that cancer is a process of clonal selection [Cairns 1975, Nowell 1976, Crespi \& Summers 2005, Merlo et al. 2006, Greaves \& Maley 2012], and game theory has often been mentioned as a relevant for cancer research [Gatenby \& Maini 2003, Merlo et al. 2006, Axelrod et al. 2006, Lambert et al. 2011, Basanta \& Deutsch 2008], the study of growth factors in the framework of evolutionary game theory is still limited. Tomlinson [1997] and Tomlinson \& Bodmer [1997] used the hawk-dove game, to explain why game theory can be used to understand conflict and cooperation between cancer cells; subsequent papers [Bach et al. 2001, 2003, Dingli et al. 2009, Basanta et al. 2008a,b, 2011, 2012, Gerstung et al 2011] have extended that model to up to 4 strategies. 2-player games, however, are not appropriate to study collective interactions and can lead to misunderstandings [Archetti \& Scheuring 2012]. The approach described here is more appropriate to the study of growth factor production in cancer cell populations. The production of growth factors, must be modelled as multi-player public goods games on planar heterogeneous networks (Voronoi networks).

Further developments that would be useful to pursue include the study of interactions on tridimensional Voronoi networks, and extensions to more complex scenarios in which the benefit function is not simply a function of the fraction of producers. In neo-angiogenesis, for example, the public good is the vascular endothelial growth factor (VEGF) produced by the cancer cells, but its diffusion range is not the same as the diffusion range of resulting benefit (the oxygen received by the cells when VEGF 
leads to the formation of a new blood vessel, which depends on the diffusion gradient of $\mathrm{O}_{2}$ ). Tumorstroma interactions, in general, depend on the diffusion of growth factors, and can be modelled using public goods games that would be more complex from the ones described here. Finally, in the Warburg effect, which also depends on diffusible public goods, one must take into account a more complex benefit function that includes self-poisoning [Archetti 2014b,c].

Other details, on the other hand, may be less crucial for modeling, either because they seem to have a relatively minor effect, like the exact shape of the diffusion gradient, or because they are arguably similar to the results we already know for regular lattices, like the effect of different update rules. Specific update rules, on the other hand, which are relevant for different types of cancer, may lead to different results for different types of cancer. Population size is also relatively unimportant, as long as it is large enough [Perc et al. 2013], although it might be interesting to analyse the dynamics in small populations (where random drift can be important) to study cooperation and competition at the inception of tumor development.

Finally, public goods games on Voronoi networks are appropriate for the analysis of other problems in biology, beyond cancer research, in ecology (interactions between sessile organisms), microbiology (production of diffusible enzymes in microbial biofilms), behavioural ecology (interactions between animals owning neighbouring territories) and any collective interaction in which players occupy the nodes of planar heterogeneous networks.

\section{Acknowledgements}

The research leading to these results has received funding from the People Programme of the European Union's Seventh Framework Programme (FP7/2007-2013) under REA grant agreement n 627816. 


\section{References}

- Aktipis CA, Kwan VSY, Johnson KA, et al. (2011) Overlooking evolution: a systematic analysis of cancer relapse and therapeutic resistance research. PLoS ONE 6: e26100.

- Allen B, Gore J, Nowak MA (2013) Spatial dilemmas of diffusible public goods. eLife 2:e01169.

- Almendro V, Marusyk A, Polyak, K. (2013) Cellular heterogeneity and molecular evolution in cancer. Annu. Rev. Pathol. 8:277-302.

- Archetti M (2013a) Evolutionary game theory of growth factor production: implications for tumour heterogeneity and resistance to therapies. British Journal of Cancer 109:1056-1062.

- Archetti M (2013b) Dynamics of growth factor production in monolayers of cancer cells and evolution of resistance to anticancer therapies. Evolutionary Applications 6:1146-1159.

- Archetti M (2013c) Evolutionarily stable anti-cancer therapies by autologous cell defection. Evolution, Medicine and Public Health 1:161-172.

- Archetti M (2014a). Stable heterogeneity for the production of diffusible factors in cell populations. PLoS One 9(9): e108526.

- Archetti M (2014b) Evolutionary dynamics of the Warburg effect: Glycolysis as a collective action problem among cancer cells. J. Theor. Biol.341:1-8.

- Archetti M (2014c) Heterogeneity and proliferation of invasive cancer subclones in game theory models of the Warburg effect. Cell Proliferation 48(2):259-269.

- Archetti M, Ferraro DA, Christofori G (2015) Heterogeneity for IGF-II production maintained by public goods dynamics in neuroendocrine pancreatic cancer. Proc. Nat. Acad. Sci. USA. 112(6):18331838.

- Archetti M, Scheuring I (2011) Coexistence of cooperation and defection in public goods games. Evolution 65:1140-1148.

- Archetti M, Scheuring I (2012) Review: game theory of public goods in one-shot social dilemmas without assortment. J. Theor. Biol. 299:9-20.

- Axelrod R, Axelrod DE, Pienta KJ (2006) Evolution of cooperation among tumor cells. Proc. Natl. Acad. Sci. USA 103:13474-13479.

- Bach LA, Bentzen S, Alsner J, Christiansen FB (2001) An evolutionary-game model of tumour cell interactions: possible relevance to gene therapy. Eur. J. Cancer 37:2116-2120.

- Bach LA, Sumpter DJT, Alsner J, Loeschke V (2003) Spatial evolutionary games of interaction among generic cancer cells. J. Theor. Med. 5:47-58.

- Barabasi, A. L. \& Albert, R. Emergence of scaling in random networks (1999) Science 286:509512.

- Basanta D, Deutsch A (2008) A game theoretical perspective on the somatic evolution of cancer. In: Selected topics on cancer modelling: genesis evolution immune competition therapy (Ed: Bellomo N, Chaplain M,De Angelis E). Birkhauser Boston.

- Basanta D, Hatzikirou H, Deutsch A (2008) Studying the emergence of invasiveness in tumours using game theory. Eur. Phys. J. 63:393-397.

- Basanta D, Simon M, Hatzikirou H, Deutsch A (2008) Evolutionary game theory elucidates the role of glycolysis in glioma progression and invasion. Cell Proliferation 41:980-987.

- Basanta D, Scott JG, Fishman MN, et al (2012) Investigating prostate cancer tumour-stroma interactions: clinical and biological insights from an evolutionary game. British Journal of Cancer 106:174-81.

- Basanta D, Scott JG, Rockne R,et al (2011) The role of IDH1 mutated tumour cells in secondary glioblastomas: an evolutionary game theoretical view. Phys Biol 8:015016.

- Beyer T, Meyer-Hermann M (2009) Multiscale modeling of cell mechanics and tissue organization. IEEE Eng Med Biol:38-45. 
- Borenstein DB, Meir Y, Shaevitz J, Wingreen NS (2013) Non-local interaction vie diffusible resource prevents coexistence of cooperators and cheaters in a lattice model. PLOS One 8:e63304.

- Cairns J (1975) Mutation selection and the natural history of cancer. Nature 255:197-200

- Cornish-Bowden A (2012) Fundamentals of Enzyme Kinetics, 4th edition; Wiley Blackwell.

- Crespi BJ, Summers K (2005) Evolutionary Biology of Cancer. Trends Ecol Evol 20:545-552.

- Csikász-Nagy A, Escudero LM, Guillaud M, et al. (2013) Cooperation and competition in the dynamics of tissue architecture during homeostasis and tumorigenesis. Seminars in Cancer Biology 23: 293-298.

- Dexter DL, Leith JT. Tumour heterogeneity and drug resistance (1986) J. Clin. Oncol. 4:244-257.

- Dingli D, Chalub FA, Santos FC, et al. (2009) Cancer phenotype as the outcome of an evolutionary game between normal and malignant cells. British Journal of Cancer 101:1130-1136.

- Gatenby RA, Maini P (2003) Cancer summed up. Nature 421:321.

- Gerstung M, Nakhoul H, Beerenwinkel N (2011) Evolutionary games with affine fitness functions: applications to cancer. Dynamic Games and Applications 1:370-385.

- Gibson WT, Gibson MC (2009) Cell Topology, Geometry, and Morphogenesis in Proliferating Epithelia. Current Topics in Developmental Biology 89:87-114.

- Greaves M, Maley CC (2012) Clonal evolution in cancer. Nature 481:306-313.

- Greenman C, Stephens P, Smith R, et al. (2007) Patterns of somatic mutation in human cancer genomes. Nature 446:153-158.

- Hanahan D, Weinberg RA (2011). Hallmarks of Cancer: The Next Generation. Cell 144(5):646674.

- Hardin, J (1968) The tragedy of the commons. Science 162:1243-1248.

- Honda H (1978) Description of cellular patterns by Dirichelet domains: the two-dimensional case. J. Theor. Biol. 72:523-543.

- Ifti M, Killingback T, Doebeli M (2004). Effects of neighbourhood size and connectivity on the spatial continuous prisoner's dilemma.J. Theor. Biol. 231:97-106.

- Jouanneau J, Moens G, Bourgeois Y, Poupon MF, Thiery JP (1994) A minority of carcinoma cells producing acidic fibroblast growth factor induces a community effect for tumor progression. Proc Natl Acad Sci USA 91:286-290.

- Jourdan M, Mahtouk K, Veyrune J, et al. (2005). Delineation of the roles of paracrine and autocrine interleukin-6 (IL-6) in myeloma cell lines in survival versus cell cycle. A possible model for the cooperation of myeloma cell growth factors. Eur. Cytokine Netw. 16:57-64.

- Karey KP, Sirbasku DA (1988) Differential responsiveness of human breast cancer cell lines MCF-7 and T47D to growth factors and 170-estradiol. Cancer Research 48:4083-4092.

- Lambert G, Estévez-Salmeron L, Oh S, et al. (2011) An analogy between the evolution of drug resistance in bacterial communities and malignant tissues. Nature Reviews Cancer 11:375-382.

- Maley CC, Galipeau PC, Finley JC, et al. (2006) Genetic clonal diversity predicts progression to esophageal adenocarcinoma. Nature Genetics 38:468-473.

- Maynard Smith J (1982) Evolution and the theory of games. Cambridge University Press.

- Meineke FA, Potten CS, Loeffler M (2001) Cell migration and organization in the intestinal crypt using a lattice-free model. Cell Proliferation 34:253.

- Merlo LMF, Pepper JW, Reid BJ, et al. (2006) Cancer as an evolutionary and ecological process. Nature Reviews Cancer 6:924-935.

- Navin N, Kendall J, Troge J, et al. (2011) Tumour evolution inferred by single-cell sequencing. Nature 472:90-94.

- Nowak MA (2006) Five rules for the evolution of cooperation. Science 314:1560-1563.

- Nowell PC (1976) The clonal evolution of tumor cell populations. Science 194:23-28. 
- Ohtsuki H, Pacheco JM, Nowak MA (2007) Evolutionary graph theory: breaking the symmetry between interaction and replacement. J. Theor. Biol. 246:681-694.

- Pepper JW (2012) Drugs that target pathogen public goods are robust against evolved drug resistance. Evolutionary Applications, 5(7):757-761.

- Perc M, Gómez-Gardeñes J, Szolnoki A, Floría LM, Moreno Y (2013) Evolutionary dynamics of group interactions on structured populations: A review. J. R. Soc. Interface 10:20120997.

- Rejniak KA, Anderson ARA (2011) Hybrid models of tumor growth. Wiley Interdisciplinary Reviews: System Biology and Medicine 3:115-125

- Rong Z,Wu Z-X (2009) Effect of the degree correlation in public goods game on scale-free networks. EPL 87:30001.

- Rong Z, Yang H-X, Wang W-X (2010) Feedback reciprocity mechanism promotes the cooperation of highly clustered scale-free networks. Phys. Rev. E 82:047101.

- Samuelson PA (1954) The pure theory of public expenditure. Review of Economics and Statistics 36: 387-389.

- Schaller G, Meyer-Hermann M (2005) Multicellular tumor spheroid in an off-lattice VoronoiDelaunay cell model. Physical Review 71:051910

- Scheuring I (2013) Diffusive public goods and coexistence of cooperators and cheaters on a 1D lattice. PLoS ONE 9(7):e100769.

- Thorne RG, Hrabetová S, Nicholson C (2004) Diffusion of epidermal growth factor in rat brain extracellular space measured by integrative optical imaging. J Neurophysiol. 92(6):3471-3481.

- Tomlinson IP (1997) Game-theory models of interactions between tumour cells. Eur. J. Cancer 33:1495-1500.

- Tomlinson IP, Bodmer WF (1997) Modelling consequences of interactions between tumour cells. British J. Cancer 75:157-160.

- Valenzano KJ, Heath-Monnig E, Tollefsen SE, et al. (1997) Biophysical and biological properties of naturally occurring high molecular weight insulin-like growth factor II variants. J. Biol. Chem. 272: 4804-4813.

- Van Leeuwen IMM, Mirams GR, Walter A, Fletcher A, Murray P, Osborne J, Varma S, Young SJ, Cooper J, Doyle B, Pitt-Francis J, Momtahan L, Pathmanathan P, Whiteley JP, Chapman SJ, Gavaghan DJ, Jensen OE, King JR, Maini PK, Waters SL, Byrne HM (2009) An integrative computational model for intestinal tissue renewal. Cell Proliferation 42(5):617-636

- Witsch E, Sela M, Yarden Y (2010) Roles for growth factors in cancer progression. Physiology. 25(2): 85-101. 
Figure 1. Voronoi graphs. a: A monolayer of cells can be described by a Voronoi tessellation of points on a plane, and the corresponding network by a planar graph (the grey lines) produced by a Delaunay triangulation of such points. An individual cell (black circle) is in direct competition with its one-step neighbours (grey circles with black edges); a group is defined by the diffusion range of the growth factor; here the diffusion range (grey cells) is $d=2$. Voronoi tessellations can be drawn on a circle (b), on a square (c) and on a sphere (d). Voronoi graphs on a sphere can be represented in two dimensions as parallelograms whose polygons on opposite edges are connected (e).

a

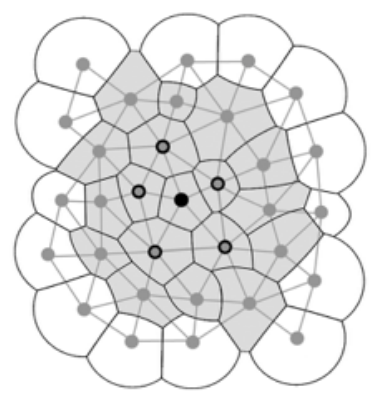

b

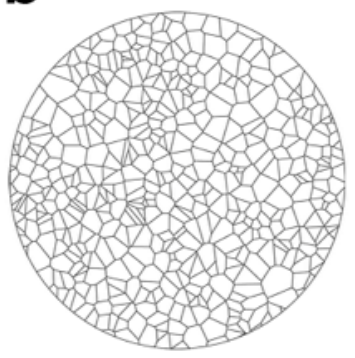

C

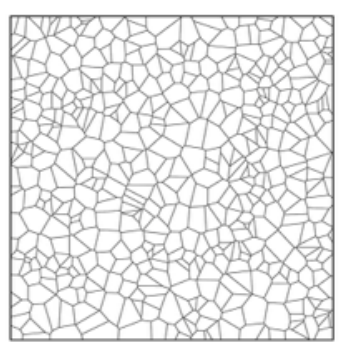

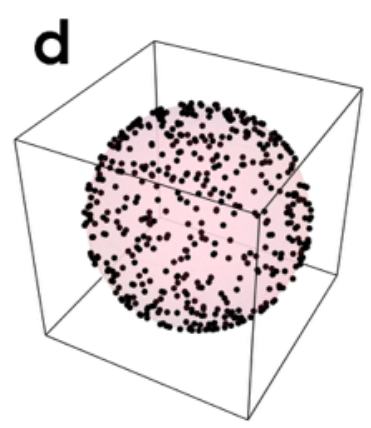

e

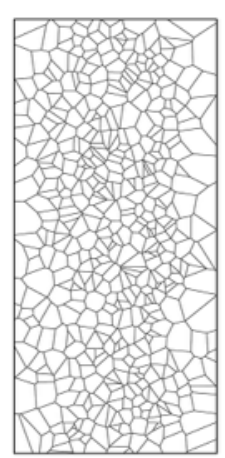


Figure 2. Difference between diffusible public goods and standard public goods on different graphs. The graphs used are Voronoi networks $(\mathbf{a}, \mathbf{b})$, regular lattices with connectivity $6(\mathbf{c}, \mathbf{d})$ and scale-free networks (e, f). Results are shown for diffusible public goods (a, c, e; diffusion range $d=2$ ), and for standard public goods $(b, d, f$; the shaded areas shows the interaction group of one of the public goods games played by the focal individual in the middle). Each cell in the contour plots shows the fraction of producers and the average fitness at equilibrium as a function of $b$ (the position of the inflection point of the benefit function) and $c$ (the cost of production).

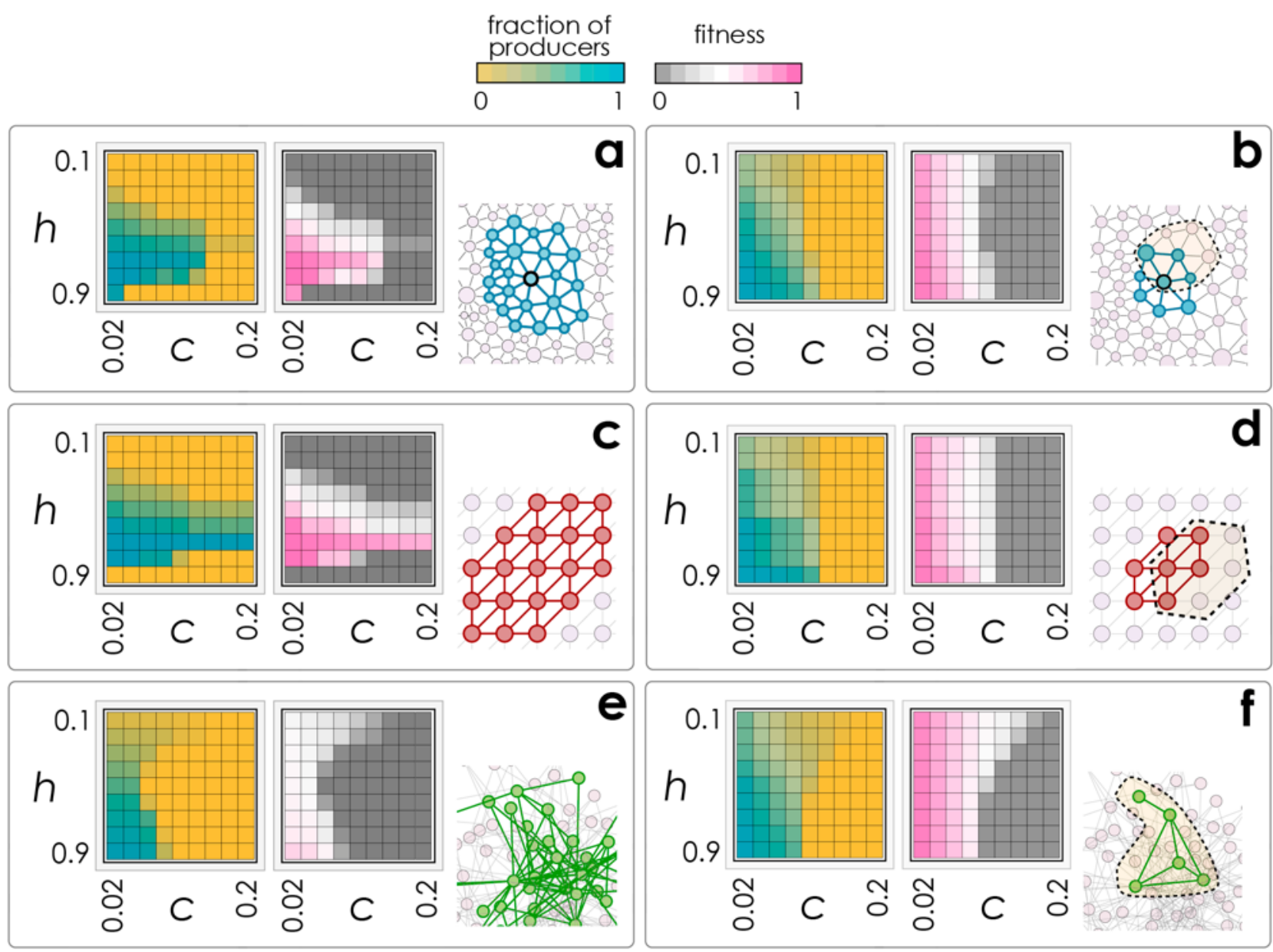


Figure 3. Group size. The average size of the update neighborhood (equivalent to the interaction neighborhood with $d=1$ ) for a Voronoi graph is $n=7$, the same value (dashed line) as the update neighborhood for a regular lattice with connectivity 6 . The smallest group size is $n=4$. The average size of the interaction neighborhood with $d=2$ is $n=20$, and with $d=5$ is $n=109$, larger than for a regular lattice with connectivity 6 (dashed lines: $n=19$ with $d=2$, and $n=91$ with $d=5$ ). Scale-free networks have many nodes with small connectivity and few nodes with much higher connectivity.

Voronoi, $d=1$

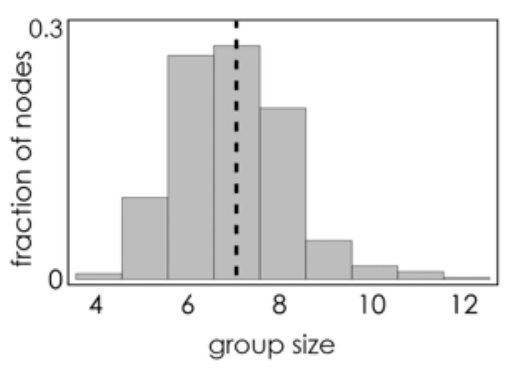

Voronoi, $d=2$

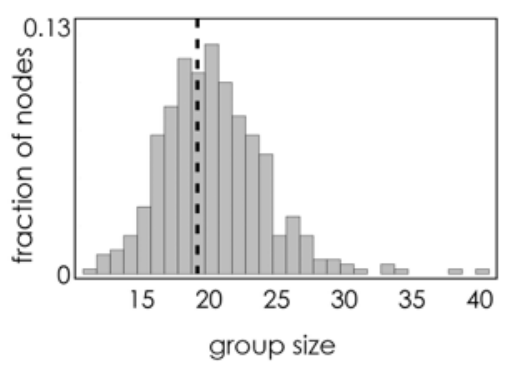

Scale-free, $d=1$

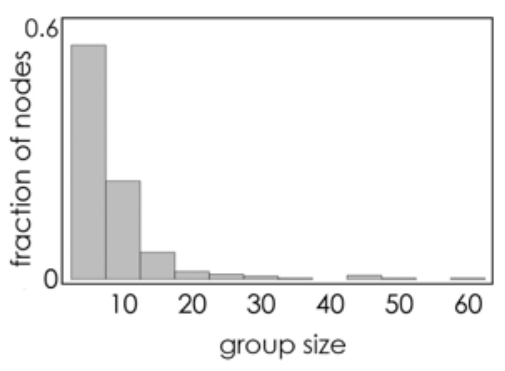

Voronoi, $d=5$

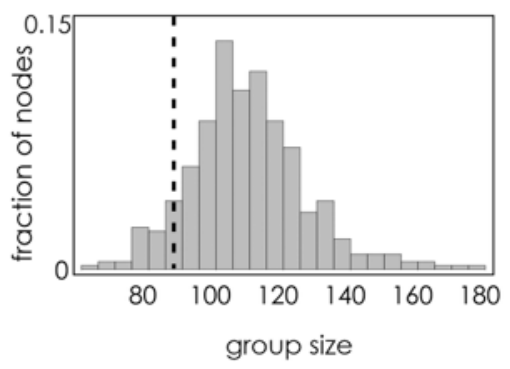


Figure 4. Effect of diffusion range, cost of production and inflection point. For different values of the diffusion range $(d)$, each cell in each contour plot shows the frequency of producers or the average fitness for a combination of $b$ (the position of the threshold) and $c$ (the cost of production) values, for $s=20$; deterministic update.

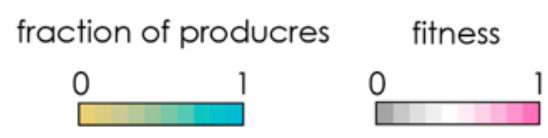

Voronoi
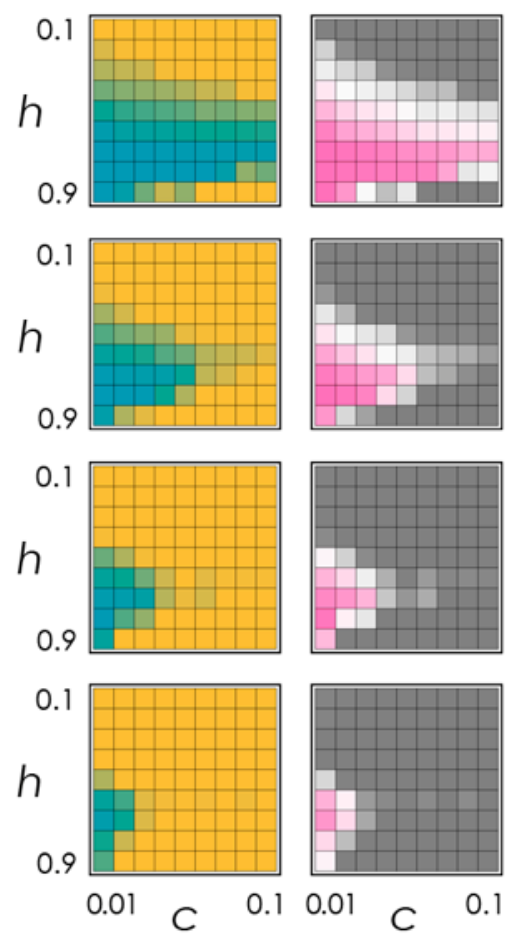

Lattice
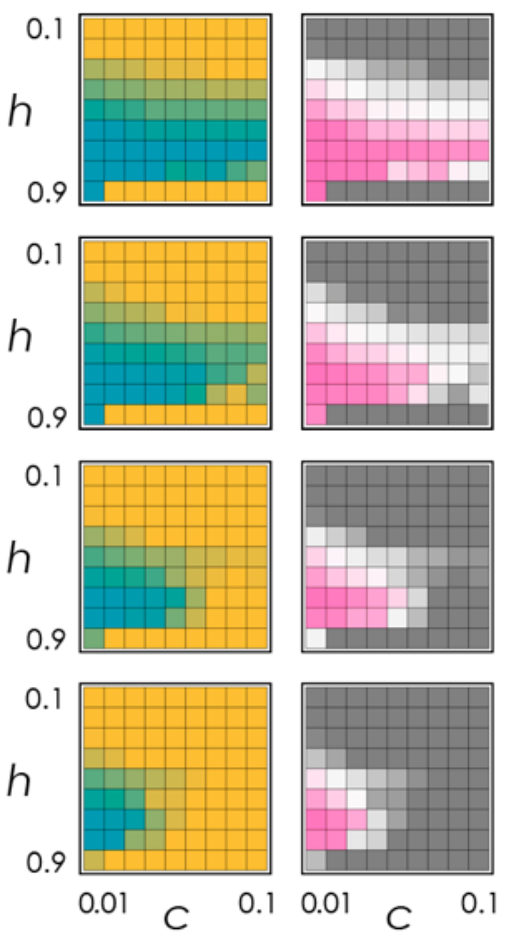

Scale free
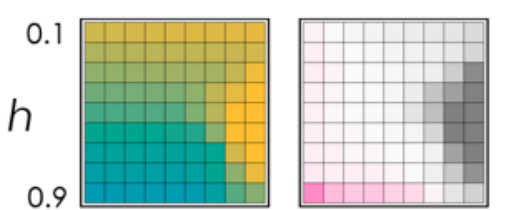

$d=2$
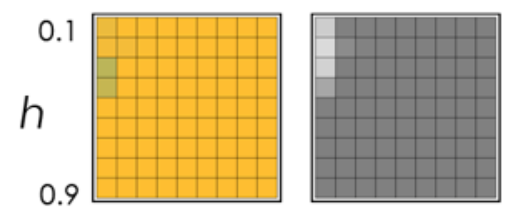

$d=3$
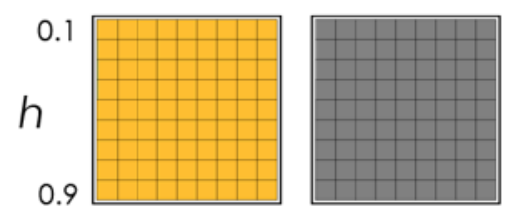

$d=4$
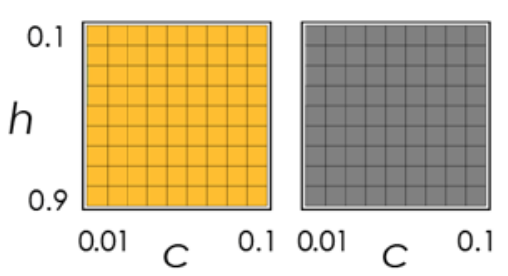

$d=5$ 
Figure 5. Effect of production cost, shape of the benefit function and update rule. Contour plots show the frequency of producers or the average fitness of the population at equilibrium in simulations with a combination of $h$ (the position of the threshold) and $c$ (the cost of production) values, for different values of $s$ (the steepness of the benefit function) and different update rules. The benefit function is shown for different values of $h$ and $s$. Voronoi graphs show snapshots of the final population with $c=0.05, b=0.5$. $d=3$.

fraction of producres

0

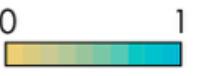

deterministic stochastic
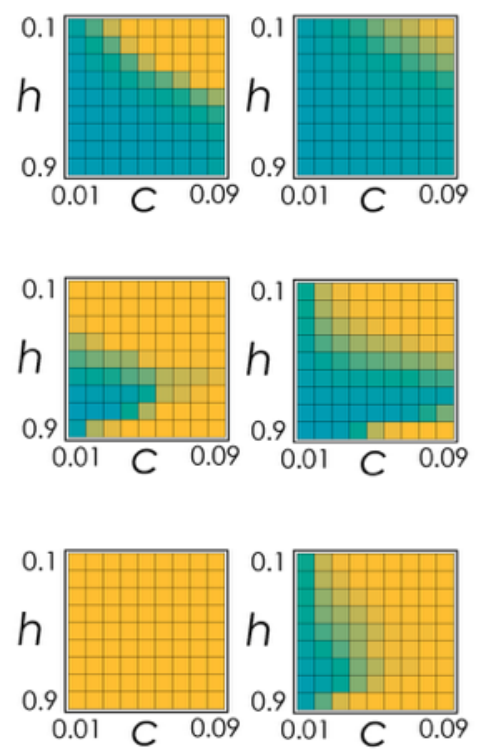

fitness
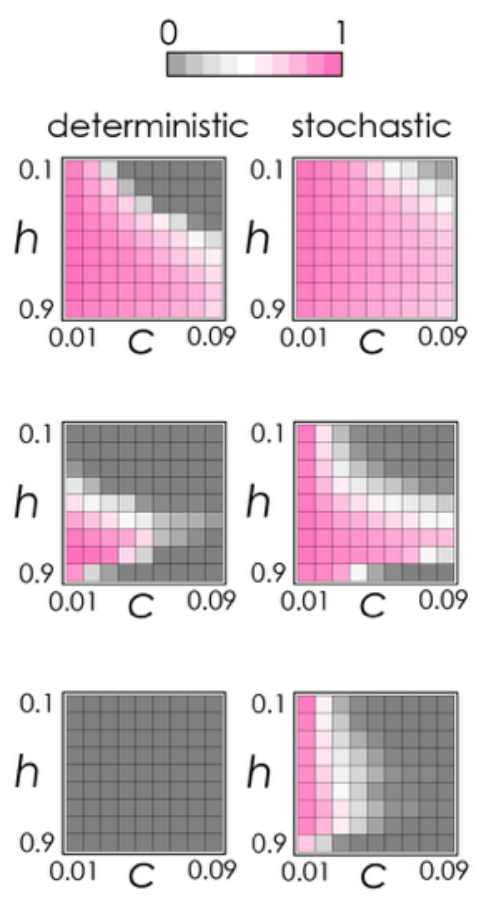
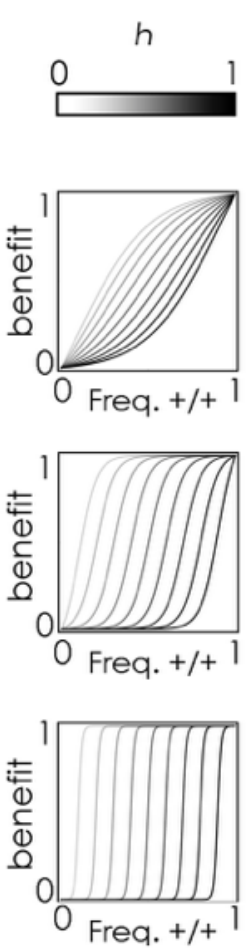
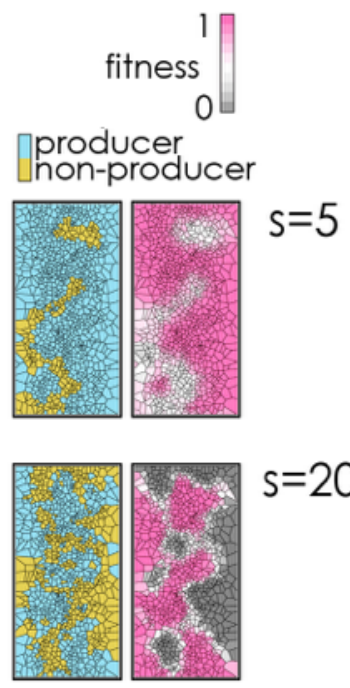

$s=20$

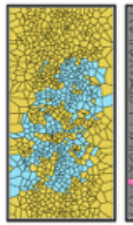

$s=100$ 
Figure 6. Changes in network structure. For different values of the diffusion range $(d)$, the Voronoi graphs show the final population structure, and the plots show the change over time of the frequency of producers, degree centrality (the number of neighbours) and closeness centrality (the shortest distance between all pairs of nodes) of the producer and non-producer subgraphs. Bold lines are the averages of 10 runs. Note that, for $d=5$, producers go extinct in some populations, which makes average degree centrality drop significantly); $c=0.01, b=0.5, s=20$, deterministic update.
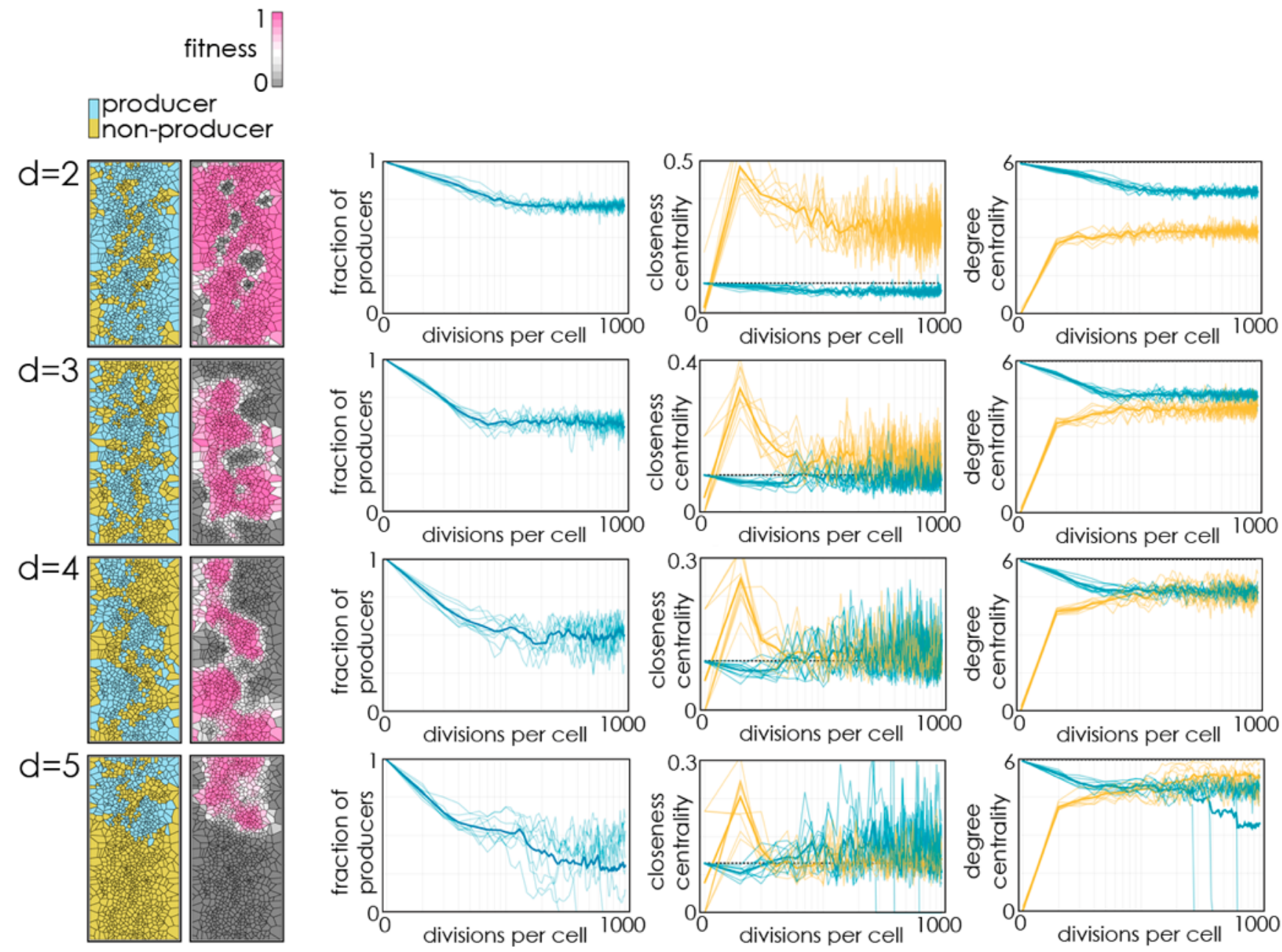
Figure 7. Equilibria with different diffusion gradients. For different benefit functions $B(x)$ and gradients of diffusion $G(i)$, the contour plots show the fraction of producers at equilibrium as a function of $h$ (the inflection point of the benefit function) and $c$ (the cost of producing the growth factor), with $s=20$. a: Fixed diffusion range with no diffusion gradient $(d=3, D=6, z=1000)$. b: Sigmoid diffusion gradient $(d=3, D=6, z=10)$. c: Linear diffusion gradient $(d=3, D=6, z=1)$. d: Concave diffusion gradient $(d=0, D=7, z=3)$.
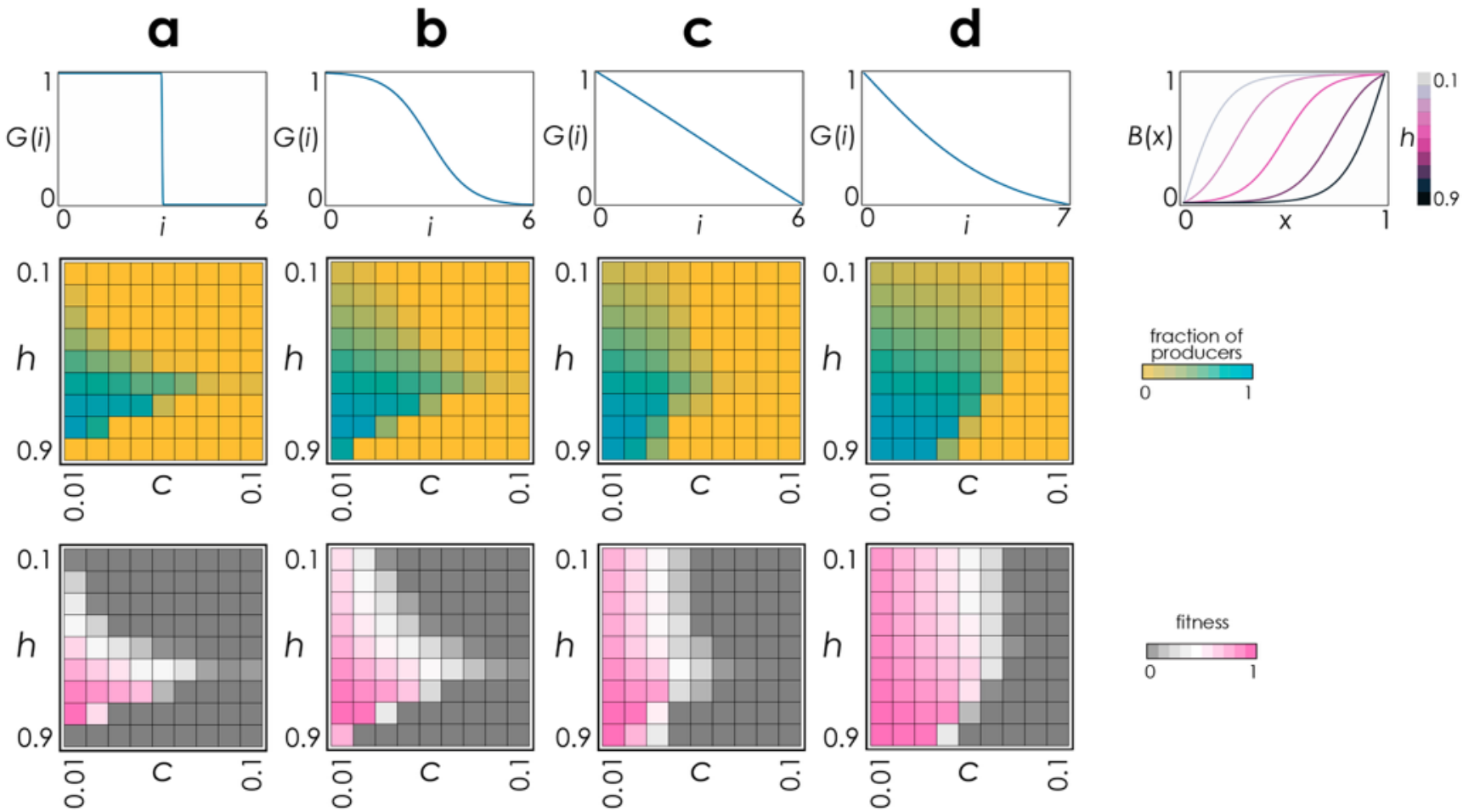
Figure 8. Changes in network structure over time, with and without diffusion gradients. a: Fixed diffusion range $(d=3, D=6, z=1000)$. b: Concave diffusion gradient $(d=3, D=6, z=1) . c=0.03, \mathrm{~s}=20$

a
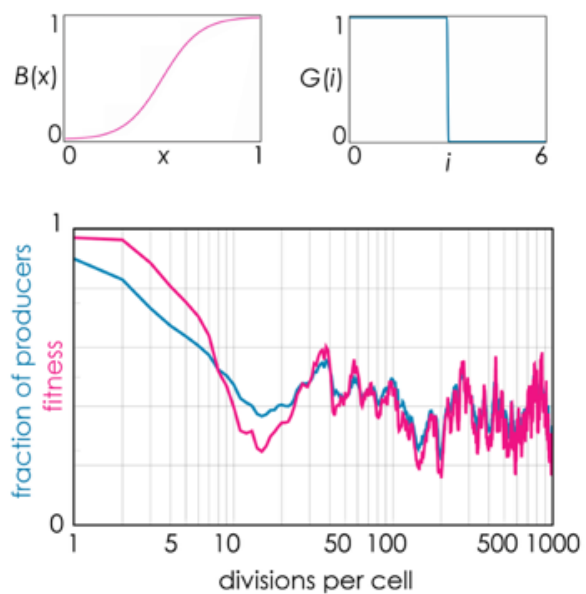

subgraph

producer $\square$ non-producer
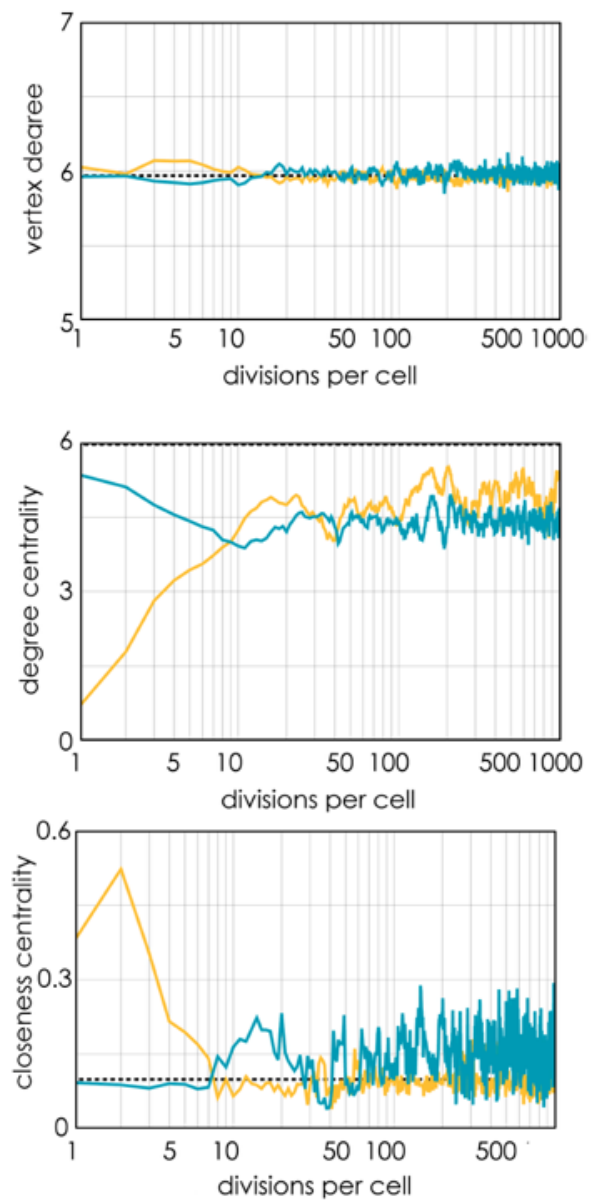
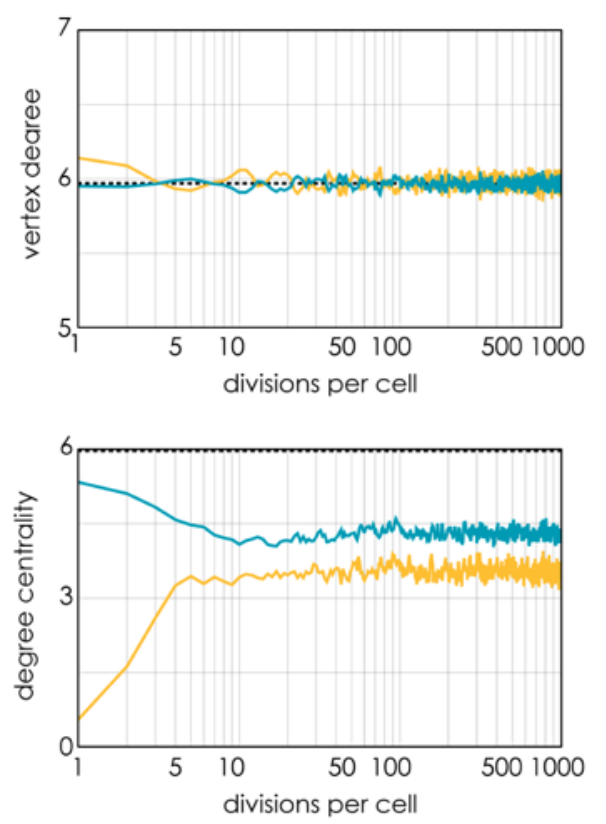

b
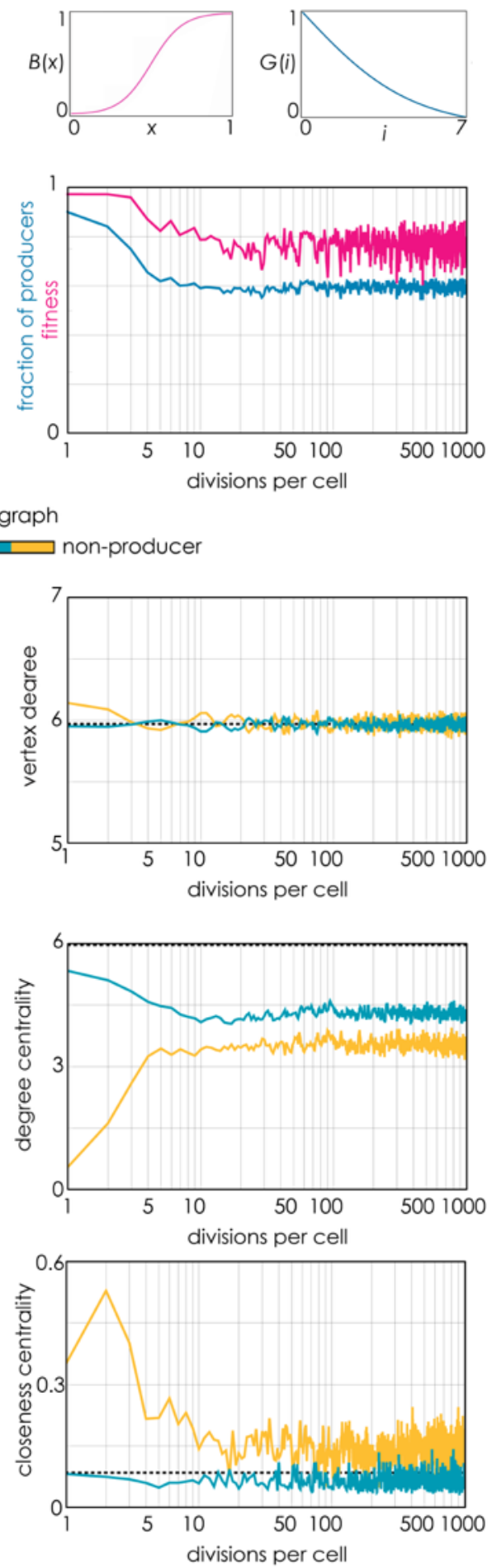
Figure 9. Evolution of resistance against therapies that target growth factors. A therapy that targets growth factors increases the amount of factors that the cells must produce to achieve the same pretreatment benefit, that is, the inflection point $h$ of the benefit function. Simulations here show the dynamics when $b$ changes from 0.5 to 0.8 at generation 1000. a: A sudden reduction in tumor growth (fitness) is immediately followed by a gradual increases in frequency of the $+/+$ type and of tumor fitness. b: Snapshots of the population at different times before and after therapy. $(d=3, c=0.02, s=10)$.

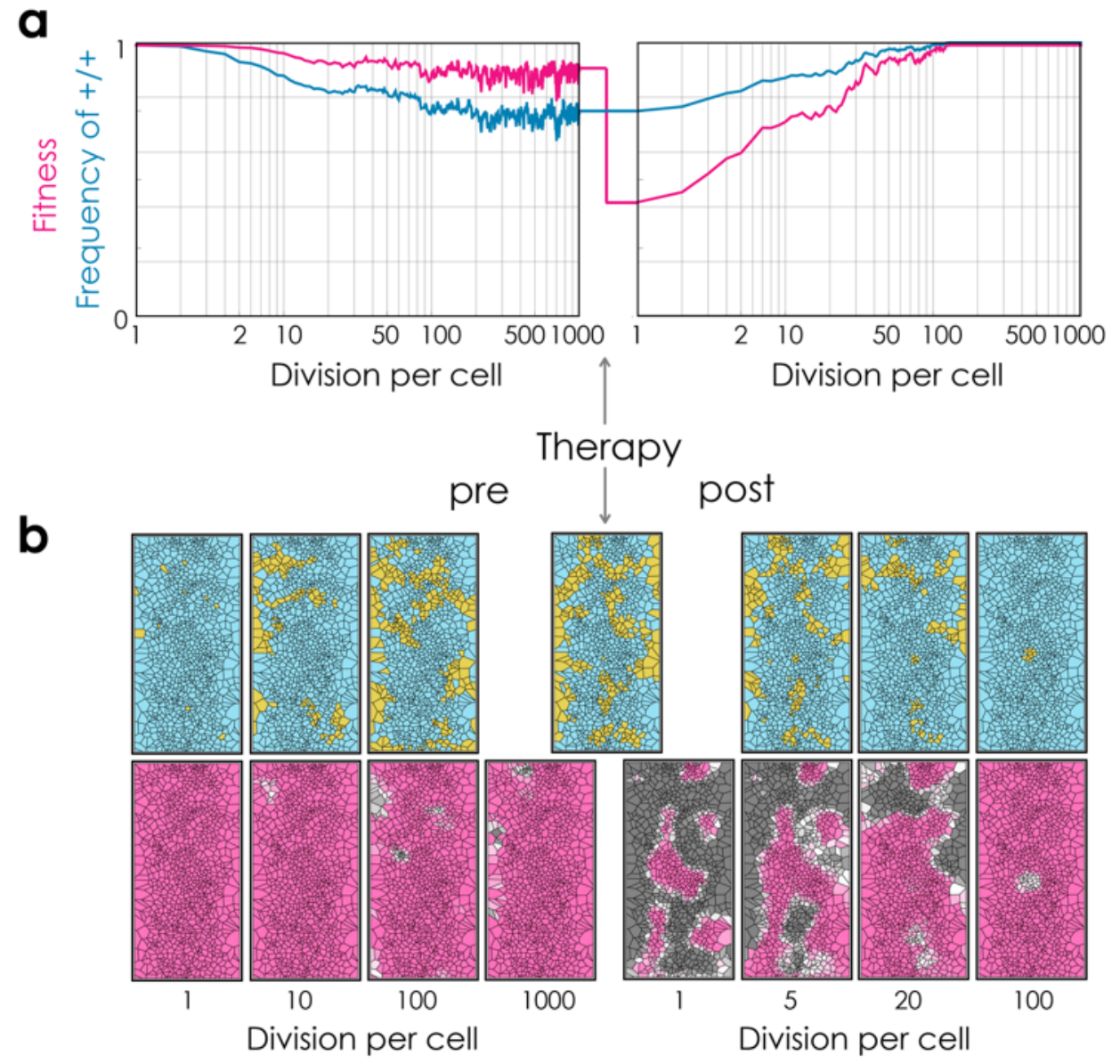


Figure 10. Effect of therapies that target growth factors. Reducing the amount of available growth factor increases the benefit threshold from $h_{1}=0.5$ to $h_{2}$. If the change from $h_{1}$ to $h_{2}$ occurs slowly (in 100 divisions per cell) tumor proliferation (fitness) does not decline because the fraction of $+/+$ cells has enough time to adjust to the new threshold, and eventually reaches a new equilibrium $\left(h_{2}=0.8\right)$, unless the reduction in circulating growth factor is extreme $\left(b_{2}=0.99\right)$. If the change is fast (10 divisions per cell) tumor fitness declines sharply and frequencies do not have enough time to adjust to the new equilibrium, which leads to a decline in tumor fitness for lack of growth factors. Bold lines are the averages of 10 simulations. $(d=3, c=0.01, s=20)$.
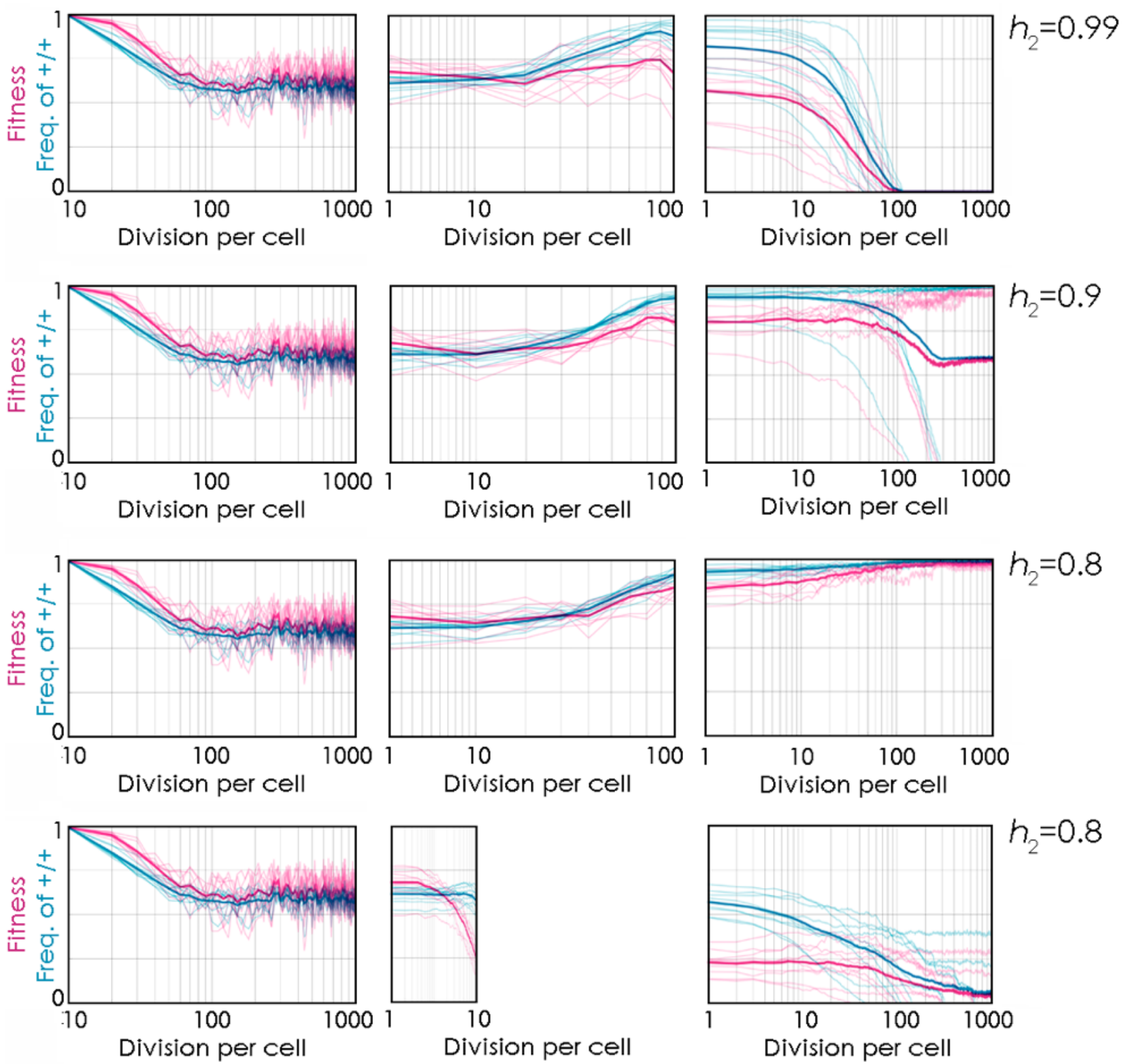